\title{
Attosecond Correlated Dynamics of Two Electrons Passing through a Transition State
}

\author{
N. Camus, ${ }^{1}$ B. Fischer, ${ }^{1}$ M. Kremer, ${ }^{1}$ V. Sharma, ${ }^{1}$ A. Rudenko, ${ }^{1,2}$ B. Bergues, ${ }^{3}$ M. Kübel,${ }^{3}$ N. G. Johnson, ${ }^{3}$ \\ M. F. Kling, ${ }^{3}$ T. Pfeifer, ${ }^{1}$ J. Ullrich, ${ }^{1,2,4}$ and R. Moshammer ${ }^{1}$ \\ ${ }^{1}$ Max-Planck-Institut für Kernphysik, 69117 Heidelberg, Germany \\ ${ }^{2}$ Max-Planck Advanced Study Group at CFEL, 22607 Hamburg, Germany \\ ${ }^{3}$ Max-Planck-Institut für Quantenoptik, 85748 Garching, Germany \\ ${ }^{4}$ Physikalisch technische Bundesanstalt, 38116 Braunschweig, Germany
}

(Received 11 August 2011; published 17 February 2012)

\begin{abstract}
The strong-field induced decay of a doubly excited, transient Coulomb complex $\mathrm{Ar}^{* *} \rightarrow \mathrm{Ar}^{2+}+2 e^{-}$is explored by tracing correlated two-electron emission in nonsequential double ionization of $\mathrm{Ar}$ as a function of the carrier-envelope phase. Using $<6 \mathrm{fs}$ pulses, electron emission is essentially confined to one optical cycle. Classical model calculations support that the intermediate Coulomb complex has lost memory of its formation dynamics and allows for a consistent, though model-dependent definition of "emission time," empowering us to trace transition-state two-electron decay dynamics with sub-fs resolution. We find a most likely emission time difference of $\sim 200 \pm 100$ as.
\end{abstract}

DOI: 10.1103/PhysRevLett.108.073003

Observing and ultimately controlling the correlated quantum motion of electrons is one of the grand challenges in contemporary research bearing exciting prospects for various applications (e.g., Cooper pair or chemical bond formation, dynamics in strongly correlated materials or quantum dots, etc.). A particularly interesting scenario is the correlated dynamics of two electrons passing through a transition state. It has been pointed out recently ([1], and references therein) that such a situation might be realized in nonsequential double ionization (NSDI) of atoms in strong laser fields. Here, at specific intensities a doubly excited intermediate Coulomb complex $A^{* *}$ can be formed that decays via $A^{* *} \rightarrow A^{++}+2 e^{-}$into the double ionization continuum by traversing a highly correlated electronic transition state - a saddle in the combined Coulomb and laser fields.

The "ultimate" experiment would be to trace the motion of the electrons as a function of time and, moreover, to possibly even control their dynamics on an attosecond time scale. This requires ultrashort laser pulses that essentially confine the electron emission to one optical cycle $(\sim 2.7 \mathrm{fs}$ for $800 \mathrm{~nm}$ ) and a field profile that can be controlled or measured on a subcycle level as recently realized by carrier-envelope phase (CEP) stabilization [2] or tagging [3]. In addition, a meaningful prescription for experimentally tracing time of two electrons in combined Coulomb and external laser fields has to be developed and verified. While at least an approximate concept, neglecting the Coulomb potential and commonly dubbed "attosecond streaking" seems to be at hand for clocking single electrons (see, e.g., $[4,5])$, a closer look onto the two-electron problem immediately questions such a method, at least if the ejection time of both electrons is similar (see also [6]).

In this Letter we report on a kinematically complete experiment on the NSDI of argon using ultrashort
PACS numbers: $32.80 . \mathrm{Rm}, 32.80 . \mathrm{Fb}, 32.90 .+\mathrm{a}, 42.50 . \mathrm{Hz}$

pulses with well-defined CEP. Choosing an intensity of $I=9 \times 10^{13} \mathrm{~W} / \mathrm{cm}^{2}$ we prepare a doubly excited $\mathrm{Ar}^{* *}$ intermediate Coulomb complex and trace its timedependent decay in the fully controlled external laser field. Within a classical model that reproduces all salient features of the experimental data, we consistently define time differences for the escape of the two electrons in terms of experimentally accessible momentum differences and, thus, can trace relative emission times and transition-state dynamics on a subcycle scale.

The essence of NSDI has been consistently explained within the recollision model $[7,8]$ where one electron is driven back to the parent ion causing ionization of the second electron in an inelastic collision (for a recent review, see [9]). The interesting case addressed here arises at a rather low intensity $\left(I=9 \times 10^{13} \mathrm{~W} / \mathrm{cm}^{2}, \lambda=\right.$ $760 \mathrm{~nm}$ ) when the recolliding electron with a maximum energy of $\sim 3.14 U_{P} \sim 15 \mathrm{eV}\left(U_{P}=I / 4 \omega^{2}\right.$ : ponderomotive potential with $\omega$ the laser frequency; atomic units are used throughout) has just sufficient energy to excite the remaining $\mathrm{Ar}^{+}$ion with field-free first excitation energies of $E_{e x}=13.5$ or $16.4 \mathrm{eV}(3 s \rightarrow 3 p$ or $3 p \rightarrow 3 d)$, respectively. Loosing essentially all of its kinetic energy during recollision, the "first" electron gets recaptured such that a doubly excited Coulomb complex is formed with large probability. It has been pointed out early on [10], that the dynamics of this doubly excited state can be treated adiabatically in good approximation due to the fast (classical) motion of the electrons as compared to the oscillation period of the laser field. Moreover, due to strong electron-electron interactions during recollision [11] it has been assumed that the intermediate state has lost any memory of its formation dynamics such that a rather generic excited Coulomb complex, a so-called compound state [10], is prepared. Using pulses of less than 6 fs this 
compound state essentially decays during one cycle, and multiple recollisions that have explained correlated electron emission for longer pulses $[12,13]$ are efficiently suppressed. Fixing the CEP in addition, guarantees identical recollision energies (within 5\% pulse-to-pulse intensity fluctuations during the experiment) and, thus, the generation of Coulomb complexes with similar excitation energies and equal saddle point potential surfaces.

The experiment has been described in detail before [14]. Briefly, the measurement was performed by using laser pulses $(\lambda=760 \mathrm{~nm})$ with duration of $6 \mathrm{fs}$ and a peak intensity of $9 \times 10^{13} \mathrm{~W} / \mathrm{cm}^{2}$. Part of the laser beam is sent into a Stereo-ATI spectrometer [3] so that the CEP of each single pulse could be tagged. The other part is focused onto a supersonic jet of argon inside the main chamber of a reaction microscope [15], which allowed us to determine the 3D momenta of all charged fragments in coincidence.

In previous work the longitudinal momentum distribution (component parallel to the laser polarization) of ions has been investigated as a function of the CEP for NSDI of Ar $[3,16]$ showing distinct asymmetries. Here, we present the complete CEP-dependent NSDI kinematics by recording the momentum vectors of the doubly charged ion and of both electrons. The experimental correlated momentum graphs are shown in Fig. 1 (upper row). The graphs are symmetrized with respect to the diagonal $P_{\| e 2}=P_{\| e 1}$.

The graph including all the CEPs (upper left panel) shows, in contrast to earlier measurements for Ar at similar intensities but longer pulses ( 220 fs [17]; $\sim 35$ fs [18]),

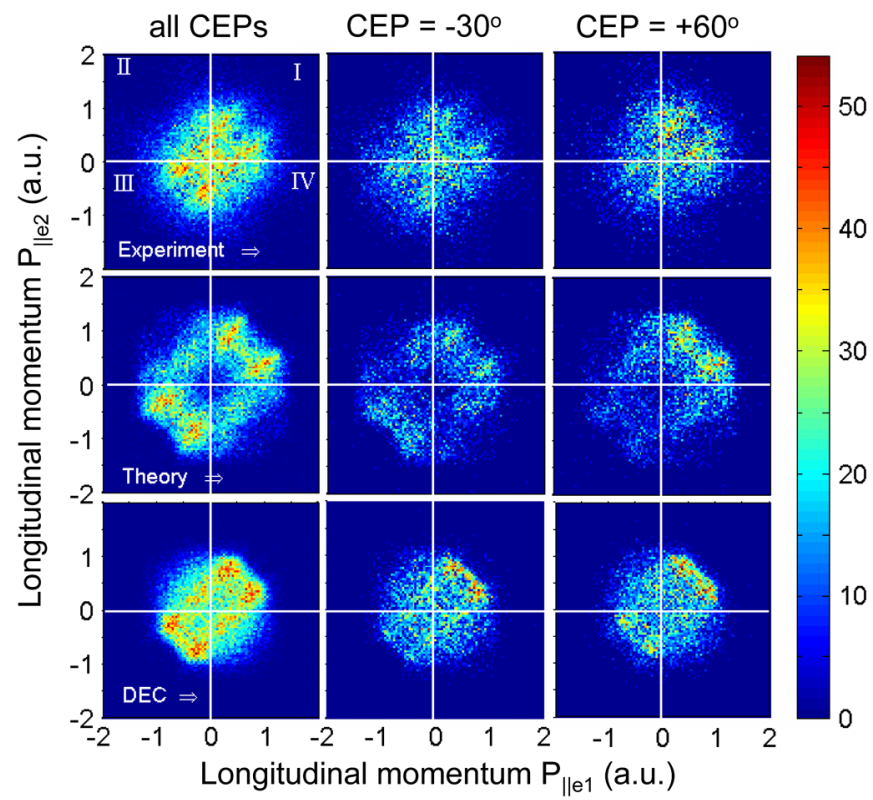

FIG. 1 (color online). Longitudinal momentum of electron 1 $\left(P_{\| e 1}\right)$ versus that of electron $2\left(P_{\| e 2}\right)$. Top row: experiment. Middle row: full classical-trajectory Monte Carlo calculation. Bottom row: compound-state model simulation (see text). Columns for CEP values as indicated. that the maximum of the distribution is not located on the diagonal, but forms two distinct lines being nearly parallel to the diagonal and located in the first and third quadrants of the plots. The pattern observed has some similarity with those measured for NSDI of He at intermediate pulse lengths where a "two-finger"-like structure was found ( $\sim 40$ fs [19], $\sim 25$ fs [20]) and it is significantly different to the "cross"-like structure that we observed recently with very short pulses ( $\sim \mathrm{fs})$ at higher intensity [21]. The dependence on the CEP is plotted (Fig. 1, upper row) for zero and maximum $\mathrm{Ar}^{2+}$ asymmetry, respectively. The left-right asymmetry is defined as $\left(N_{l}-N_{r}\right) /\left(N_{l}+N_{r}\right)$ with $N_{l,(r)}$ the number of $\mathrm{Ar}^{2+}$ ions being emitted to the left (right) along the laser polarization direction. The CEP has a distinct influence on the electron dynamics in the recollision process and, thus, on their final momenta. This can be clearly observed: the distribution displays a center of gravity equal to zero for a CEP of $\varphi=-30^{\circ}$ (and $\varphi=$ $150^{\circ}$ ) and it shifts with the CEP along the $P_{\| e 2}=P_{\| e 1}$ diagonal. For $\varphi=60^{\circ}$ (and $\varphi=-120^{\circ}$ ) we observe a maximum asymmetry in the emission direction of the electrons. Since the CEP in the experiment is only determined relatively by tagging the electron asymmetry with the Stereo-ATI, the absolute CEP value is chosen by comparison with theory (lower two rows in Fig. 1).

In order to better understand and consistently analyze the electron dynamics we have performed two different simulations. In a first calculation (Fig. 1, middle row) we adopt a semiclassical approach as proposed, e.g., by [22]. We assume the first electron to be tunnel ionized with a probability according to the Landau rate [23] by placing it at the outer edge of the Coulomb barrier with zero longitudinal velocity and a Gaussian distribution centered at zero for the transverse velocity [24]. The second electron is placed on an elliptic orbit in the screened Coulomb potential of the doubly charged ion resembling the microcanonical distribution [25] and bound with the $\mathrm{Ar}^{+}$ionization potential $(27.6 \mathrm{eV})$. From then on both electrons and the ionic core are propagated fully classically in 3D considering all mutual Coulomb interactions between the charged particles and their interactions with the electric laser field $F(t)=F_{0} \cos ^{2}\left(\pi t / T_{p}\right) \cos (\omega t+\varphi) \quad\left(T_{p}=\right.$ pulse length, $\varphi=\mathrm{CEP})$. At the present intensity direct impact ionization of the second electron is essentially impossible. Moreover, it is found that the RESI ("recollision excitation plus subsequent ionization" [26]) contribution to double ionization via tunneling of the excited electron is very small, less than $10 \%$ of all ionization events [27], such that we can neglect this process in good approximation. Thus, the dominant contribution to NSDI is the formation of a doubly excited state from which, subsequently, both electrons escape over the laser-field suppressed barrier in a highly correlated manner. In total about $5 \times 10^{6}$ trajectories have been calculated that resulted in some ten thousand double ionization events. 
Comparing the model results (Fig. 1, middle row) with the experimental data averaged over all CEPs we find good agreement. The discrepancies at small momenta $\left(P_{\| e 2}=\right.$ $\left.P_{\| e 1}=0\right)$ might be due to two effects that are not included in the model: electron tunneling after recollision and intensity averaging over the focal volume in the experiment. Also the variation of the correlated momentum spectra with the CEP resembles the experimental findings. The fact that the theoretical asymmetry is more pronounced we again attribute to the neglect of focal volume effects in the model.

In order to illustrate the different steps in theory we present the evolution of the binding energies of both electrons relative to the ion as a function of time for two specific, nevertheless typical NSDI events in Fig. 2. As described before, we observe the creation of a doubly excited state at a quite well-defined instant in time (gray shaded areas). The two electrons are then freed into the continuum (energy larger than zero) at one of the next electric field maxima. According to our calculations in $97 \%$ of all double ionization events a doubly exited state is formed for NSDI under present conditions.

A "true" compound state as known, e.g., from nuclear excited states ideally has lost any memory about its creation process. To investigate this phenomenon theoretically we generated a classical doubly excited state and followed its decay along the three dimensional saddle formed by the Coulomb potential and the laser field. At the time of

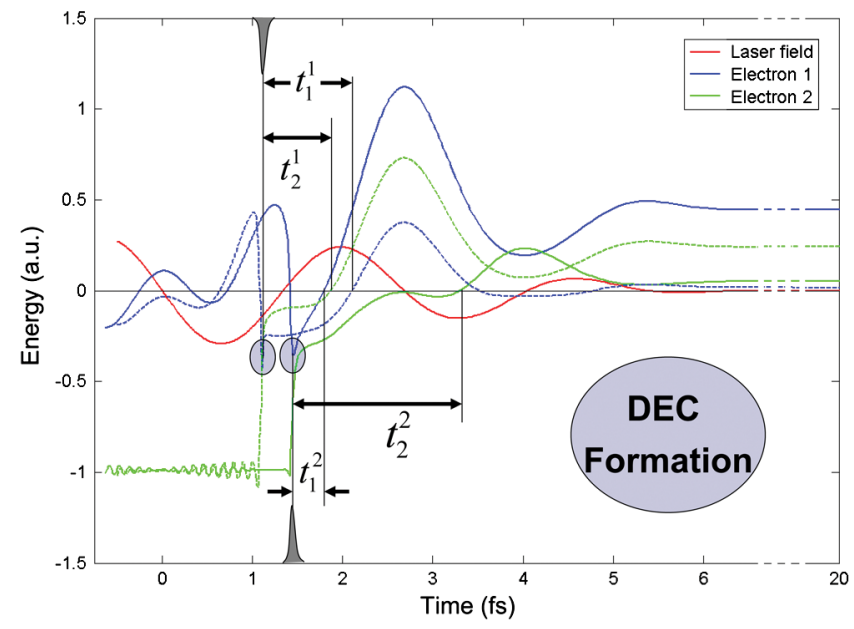

FIG. 2 (color online). Energy of electron 1 and 2 versus time for two sample trajectories (solid and dashed lines) of the full calculation. Electron 1 tunnels close to a maximum of the electric field and excites the second electron during recollision about 1.5 half cycles later (gray shaded areas). Because of its low recollision energy electron 1 becomes bound during this process and a doubly exited complex (DEC) is created. The electrons are released into the continuum at one of the next electric field maxima via classical "over the barrier ionization." For each event $i$ the ionization times $t_{1}^{i}$ and $t_{2}^{i}$ of electrons 1 and 2, respectively, are defined as the instants when their energy (ion potential plus kinetic) becomes positive. recollision we consider an atom with two excited electrons. We choose one specific realization of a nonautoionizing classical two-electron state by placing the electrons diametrically opposite to each other on identical elliptic orbits with identical angular velocities. The resulting correlated graph for a starting binding energy of 0.3 a.u. for each excited electron is shown in Fig. 1. Again the essential features are in good agreement with the experimental results and the full simulation (middle row). However, the graphs with different CEPs show a slight discrepancy: for a phase of $\varphi=-30^{\circ}$, the center of the distribution is not located at $(0,0)$ in contrast with the full calculation. This finding shows that even though the major characteristics are reproduced, the first step of the process cannot entirely be neglected. It influences the binding-energy distribution of the two electrons in the doubly excited complex (DEC) and thus their dynamics.

How do the correlated electrons leave the transition state and can we "observe" them by tracing their respective "crossing times," i.e., the instant of ionization? To this end we have to define this time that would, for a free electron, simply correspond to the instant when its total energy (kinetic plus potential) turns positive. For two active electrons including the laser field this is not a trivial task. Moreover, even a single electron considered "free" at a given time might evolve to be bound again at the end of the laser pulse, which is sometimes referred to as "frustrated ionization" [28], a process usually not considered and not consistently discussed in so-called "streaking scenarios." Whereas we cannot provide a general solution to this problem we may define consistent "ionization time differences between the two electrons" $\Delta T$ within the classical model and relate those to observable momentum differences between both electrons. We inspect $\Delta T=$ $\left|t_{2}^{i}-t_{1}^{i}\right|$ for each individual event $i$ and define the ionization time $t_{1,2}^{i}$ as the instant when the energy of one electron becomes positive for the first time. In analogy to others $[12,24]$, the single electron energy is calculated considering the Coulomb interaction with the ion, neglecting both the electron-electron repulsion and the interaction with the laser field. In Fig. 3 the $\Delta T$ distribution for the DEC calculation is presented. The main peak at $\Delta T=0$ containing $80 \%$ of all events corresponds to scenarios when the electrons are ionized in the same half cycle.

Whether we can experimentally trace in time the correlated ionization of two electrons out of a transition state decisively depends on whether the theoretically defined $\Delta T$ can be quantitatively related to an experimentally observable quantity. In the streaking scenario the observable is the momentum shift imposed onto the electron as a function of the release time $t$, which is equal to the instantaneous laser-field vector potential $p_{\text {streak }}(t)=-A(t)$. We thus inspect the correlated momentum plots for various $\Delta T$ (Fig. 3, inset panels) and find a striking general difference. Whereas events from the main peak (ionization within one 


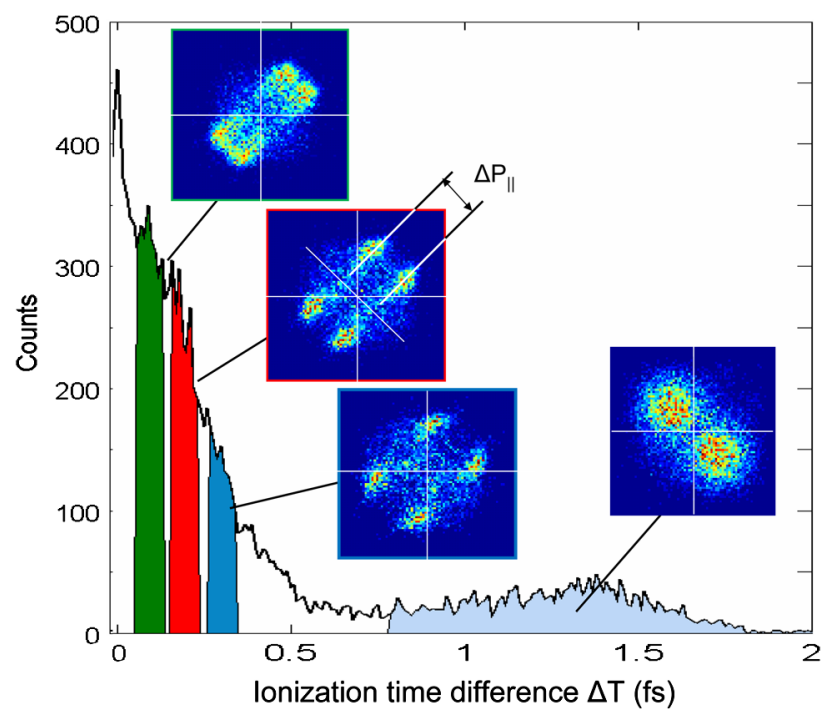

FIG. 3 (color online). Distribution of the ionization time difference $\Delta T=\left|t_{2}^{i}-t_{1}^{i}\right|$ for the DEC calculation (averaged over all CEPs). Insets: correlation maps (the momentum scale ranges from -1.5 to $1.5 \mathrm{au}$ ) obtained when selecting specific time differences $\Delta T$ as indicated.

half cycle) predominantly show correlated emission into the same hemisphere those in the secondary peak at $\Delta T \approx$ $1.3 \mathrm{fs}$ (ionization in successive half-cycles) are strongly anticorrelated because the sign change in the laser electric field drives the electrons into opposite hemispheres. Moreover, and most interestingly, for events resulting from ionization of both electrons within the same half cycle we find a clear correlation between $\Delta T$ and the longitudinal momentum difference between the two electrons $\Delta P_{\|}=P_{\| e 1}-P_{\| e 2}$ along the $P_{\| e 2}=-P_{\| e 1}$ diagonal.

In Fig. 4(a) we plot $\Delta T$ versus $\Delta P_{\|}$for the DEC as well as for the full calculation in order to investigate the influence of the first steps. Here, for the full calculation, electrons 1 and 2 are the recolliding and the bound electron, respectively. We find a clear and unique correlation for $\Delta P_{\|} \leq \approx 1.3$ a.u. [shaded area in Fig. 4(a)] for the DEC calculation, where the electrons cannot be distinguished, as well as for the full model even though with slightly different absolute time differences of $<100$ as depending on whether electron 1 or 2 is emitted first hinting towards some "memory effect." Clearly, the $\Delta P_{\|} \leftrightarrow \Delta T$ relationship is not ideal and an error bar has been extracted from the simulations indicated by the shaded area. Nevertheless, for small $\Delta P_{\|}$time differences are determined with a FWHM of about \pm 50 as.

Based on these findings we compare experimental and simulated $\Delta P_{\|}$values and relate them to $\Delta T$. In Figs. 4(b) and $4(\mathrm{c}), \Delta P_{\|}$distributions are presented for the experiment and the DEC calculation. Here, we illustrate again the behavior observed before in Fig. 3, namely, decreasing $\Delta P_{\|}$with decreasing $\Delta T$. A striking agreement between experimental and simulated $\Delta P_{\|}$distributions is observed
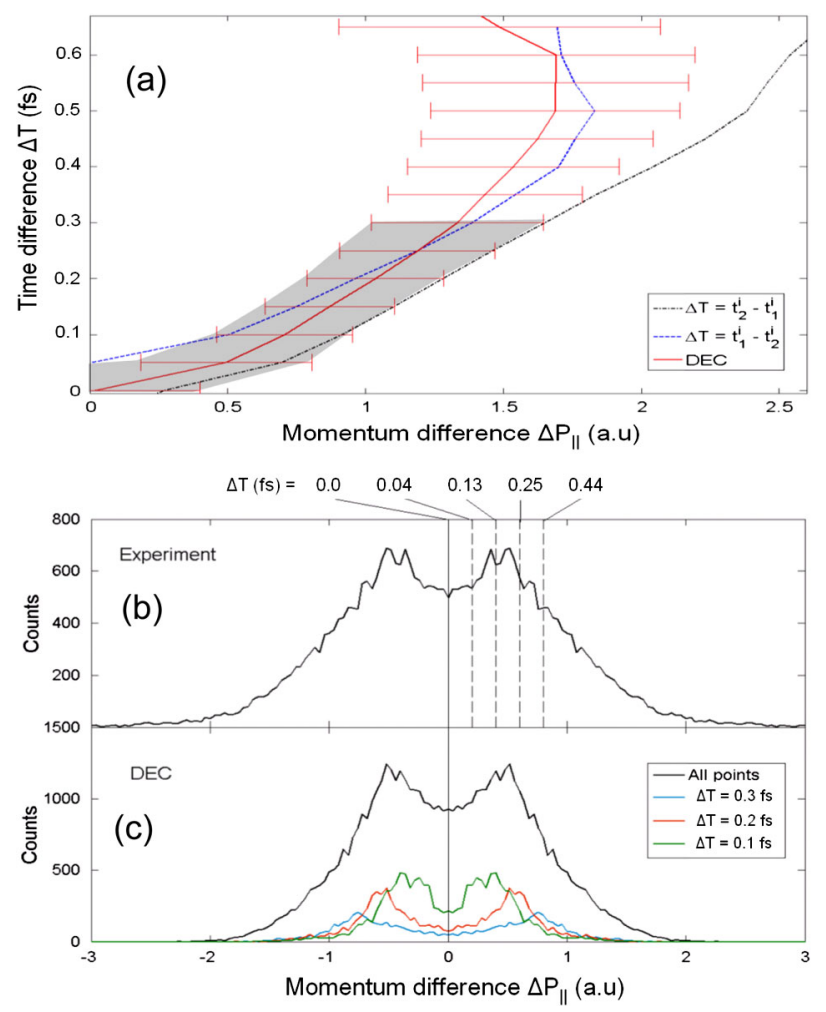

FIG. 4 (color online). (a) Ionization time difference $\Delta T$ as a function of momentum difference $\Delta P_{\|}$(see also Fig. 3) for the case of the DEC as well as the full calculation. (b) Experimental distribution of the electron momentum difference $\Delta P_{\|}$averaged over all CEPs. (c) Same as (b) obtained with the DEC calculation without any restriction on $\Delta T$ and for selected values of $\Delta T$ (according to the correlation maps shown in Fig. 3). On the upper ordinate the time scale $\Delta T$ is provided as extracted from the simulation.

with a minimum at $\Delta P_{\|} \approx \Delta T=0$ and a most likely time difference between the two electrons for leaving the transition state of $200 \pm 100$ as.

In conclusion, we provide evidence that a doubly excited complex is formed in NSDI of Ar via electron recollision below the direct recollision-ionization threshold energy. Within a classical simulation we demonstrate that this complex has essentially lost memory of its creation. We consistently relate measured momentum differences between the two electrons to time differences and, thus, follow the decay of the doubly excited complex on a subfs time scale. This study represents a first step towards tracing in time the ultrafast correlated motion of two electrons out of a bound state.

A. R. acknowledges support from the Max-Planck Advanced Study Group at CFEL and T.P thanks the MPRG program of the Max-Planck Society.

[1] B. Eckhardt et al., Chem. Phys. 370, 168 (2010).

[2] A. Baltuska et al., Nature (London) 421, 611 (2003). 
[3] N. G. Johnson et al., Phys. Rev. A 83, 013412 (2011).

[4] M. Schultze et al., Science 328, 1658 (2010).

[5] A. N. Pffeifer et al., Nature Phys. 7, 428 (2011).

[6] D. I. Bondar, W. K. Liu, and M. Y. Ivanov, Phys. Rev. A 79, 023417 (2009).

[7] P. B. Corkum, Phys. Rev. Lett. 71, 1994 (1993).

[8] M. Y. Kuchiev, JETP Lett. 45, 404 (1987).

[9] C. Figueira de Morisson Faria and X. Liu, J. Mod. Opt. 58, 1076 (2011).

[10] K. Sacha and B. Eckhardt, Phys. Rev. A 63, 043414 (2001).

[11] M. Weckenbrock et al., Phys. Rev. Lett. 91, 123004 (2003).

[12] P. J. Ho et al., Phys. Rev. Lett. 94, 093002 (2005).

[13] A. Rudenko et al., Phys. Rev. A 78, 015403 (2008).

[14] V.L.B. de Jesus et al., J. Electron Spectrosc. Relat. Phenom. 141, 127 (2004).
[15] J. Ullrich et al., Rep. Prog. Phys. 66, 1463 (2003).

[16] X. Liu et al., Phys. Rev. Lett. 93, 263001 (2004).

[17] Th. Weber et al., Nature (London) 405, 658 (2000).

[18] E. Eremina et al., J. Phys. B 36, 3269 (2003).

[19] A. Staudte et al., Phys. Rev. Lett. 99, 263002 (2007).

[20] A. Rudenko et al., Phys. Rev. Lett. 99, 263003 (2007).

[21] B. Bergues et al. (to be published).

[22] G. G. Xin, D. F. Ye, and J. Liu, Phys. Rev. A 82, 063423 (2010).

[23] L.D. Landau and E. M. Lifshitz, Quantum Mechanics (Pergamon, Oxford, 1965).

[24] J. Liu et al., Phys. Rev. Lett. 99, 013003 (2007).

[25] J. G. Leopold and I. C. Percival, J. Phys. B 12, 709 (1979).

[26] B. Feuerstein et al., Phys. Rev. Lett. 87, 043003 (2001).

[27] D. F. Ye and J. Liu (private communication).

[28] T. Nubbemeyer et al., Phys. Rev. Lett. 101, 233001 (2008). 